\title{
UltraSail - Ultra-Lightweight Solar Sail Concept
}

\author{
Rodney L. Burton, ${ }^{*}$ Victoria L. Coverstone, ${ }^{\dagger}$ Jennifer Hargens-Rysanek, ${ }^{\ddagger}$ Kevin M. Ertmer ${ }^{\S}$, and Thierry Botter ${ }^{* *}$ \\ University of Illinois, Urbana, IL 61801 \\ Gabriel Benavides, ${ }^{\dagger+}$ Byoungsam Woo, ${ }^{\ddagger \ddagger}$ and David L. Carroll
CU Aerospace, Champaign, $I L, 61820$ \\ Paul A. Gierow ${ }^{* * *}$ and Greg Farmer ${ }^{\dagger+\dagger}$ \\ SRS Technologies, Inc., Huntsville, AL, 35806 \\ Joe Cardin ${ }^{\ddagger \ddagger}$ \\ Vacco Industries, South El Monte, CA 91733
}

\begin{abstract}
UltraSail is a next-generation high-risk, high-payoff sail system for the launch, deployment, stabilization and control of very large $\left(\mathrm{km}^{2}\right.$ class) solar sails enabling high payload mass fractions for high $\Delta V$. UltraSail is an innovative, non-traditional approach to propulsion technology achieved by combining propulsion and control systems developed for formation-flying micro-satellites with an innovative solar sail architecture to achieve controllable sail areas approaching $1 \mathrm{~km}^{2}$, sail subsystem area densities approaching $1 \mathrm{~g} / \mathrm{m}^{2}$, and thrust levels many times those of ion thrusters used for comparable deep space missions. UltraSail can achieve outer planetary rendezvous, a deep space capability now reserved for high-mass nuclear and chemical systems. One of the primary innovations is the near-elimination of sail supporting structures by attaching each blade tip to a formation-flying micro-satellite which deploys the sail, and then articulates the sail to provide attitude control, including spin stabilization and precession of the spin axis. These tip micro-satellites are controlled by 3-axis micro-thruster propulsion and an on-board metrology system. It is shown that an optimum spin rate exists which maximizes payload mass.
\end{abstract}

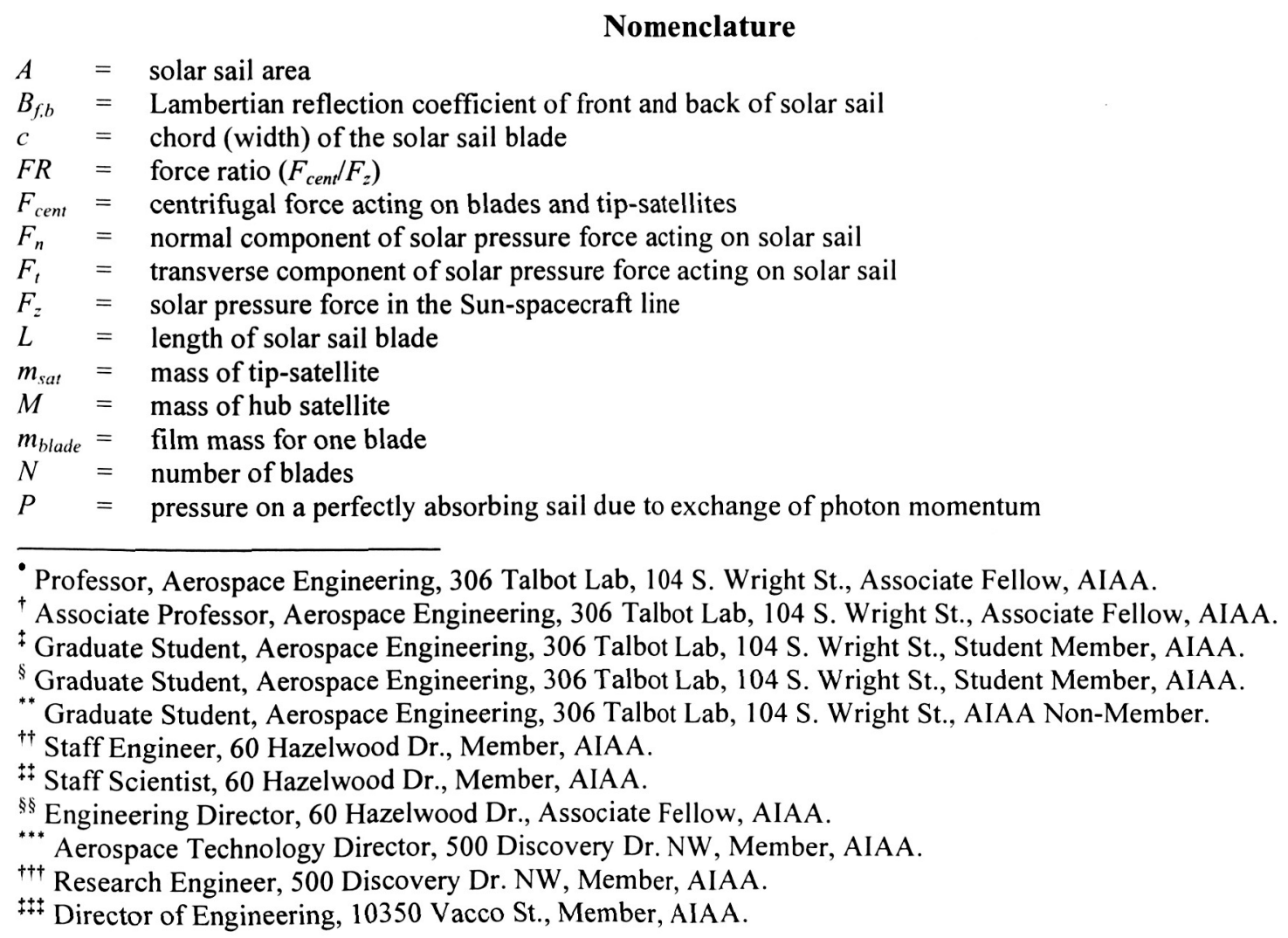




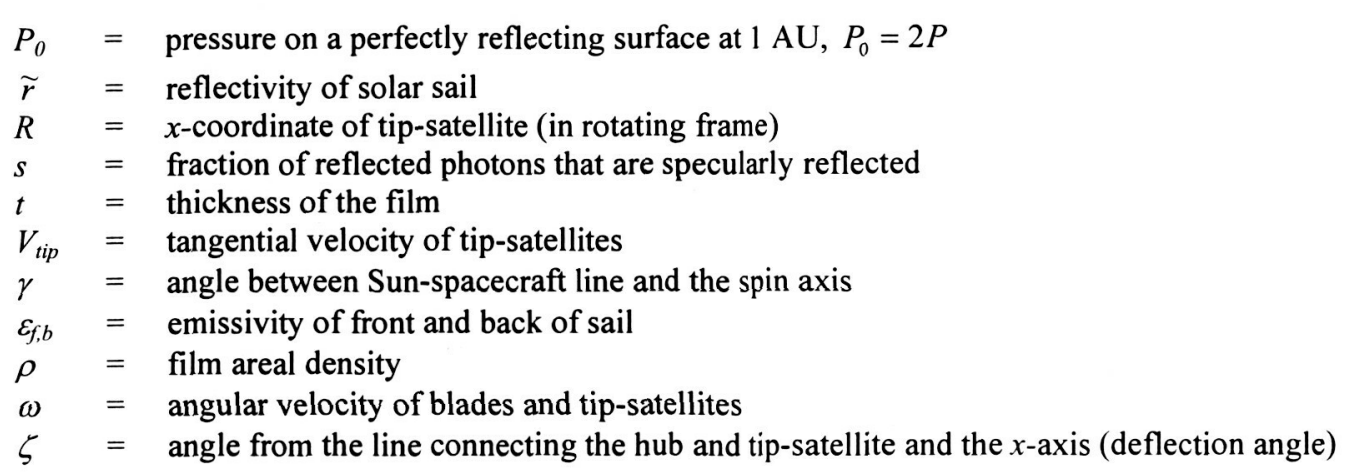

\section{Introduction}

$\mathrm{T}$ HE goal of any spacecraft is to deliver as much payload as possible within a given timeto a given destination with minimum expenditure of propellant. This is done by minimizing the amount of mass needed for components in excess of the payload. Solar sails could potentially combat this problem, since the propulsion system consists only of an extremely lightweight thin film, rather than massive propellant and the associated tankage and apparatus. Recently, there has been a reemergence of interest in solar sails in the National Aeronautics and Space Administration (NASA) community as they are becoming an increasingly viable option for fulfilling this goal. NASA's In-Space Propulsion Technology Program has identified solar sailing as one of its key propulsion technology priorities and has been leading the way to establish these technologies. ${ }^{1}$ Some of the nearer-term solar sail projects that are being proposed are the Solar Polar Imager (SPI), the L1 Diamond and the Particle Acceleration Solar Orbiter (PASO). ${ }^{2}$ The SPI mission objective is to measure the Sun's magnetic field, polar irradiance, and coronal mass ejections. The L1 Diamond mission is a constellation of four spacecraft concurrently and cooperatively gathering data. The L1 constellation of spacecraft will hover on the Sun-ward side of the Earth-Sun L1 libration point. The objective of this mission is to measure the properties of solar-wind turbulence as a function of space and time. The PASO mission includes transferring a payload of scientific instruments to a very close solar orbit. These instruments will capture high resolution images of high energy solar flares, thereby determining composition of these flares. These missions are best performed with solar sails because of the high energy requirements.

One of the acknowledged problems with solar sails has been the large sail area needed for adequate propulsion. Solar sail materials have matured to a point that they can theoretically perform solar sail missions, so the problem of deployment and rigidity of the system needs to be addressed. Conventional solar-sailing spacecraft designs utilize large booms to deploy and support the thin film of reflective material. These boom-deployed schemes are limited in size by the penalties involved in the boom mass compared to the sail area, specifically Euler column bending of the booms. It logically follows that eliminating the booms could provide larger sail area, higher thrust, and lower areal density, resulting in higher acceleration and higher payload mass fractions. Removing these support structures is at the core of the UltraSail concept.

In the UltraSail system, there is a central hub where the payload would reside. Attached to this hub would be several "blades" of solar sail film material that would unroll from a storage mandrel with the help of a tip microsatellite that is attached to the end of each blade. The baseline UltraSail design has four blades composed of a micron-thick reflection-coated polyimide film. During the deployment of the blades, the formation flying tip satellites spin up the entire system to create a spin-stabilized, controllable solar sail system, with a large sail area, conceivably up to $1 \mathrm{~km}^{2}$ in area. (See Figure 1) 


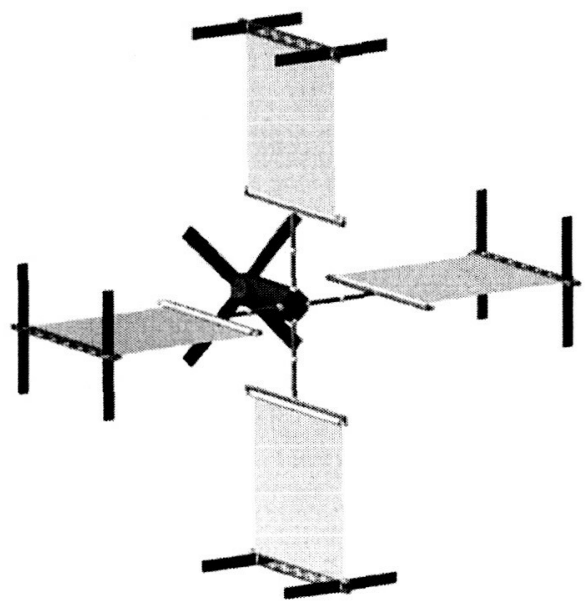

Figure 1. UltraSail Conceptual Drawing.

The tip-satellite, a lightweight beam truss with onboard propulsion, performs the following functions:

1. A stable, stiff attachment point for the film end.

2. Bus for propellant, solar panels, thrusters, and a metrology system.

3. On-board thrust to initiate film roll-out and initial blade spin.

4. Satellite metrology system to provide blade tip position, velocity and acceleration.

5. Twists the blades to induce torques for spin-stabilization, using pitch thrusters.

6. Satellite centrifugal force flattens blade to increase photon thrust.

7. Provides continuous plane-change control for Sun-orientation of spacecraft spin axis.

8. Blade camber control to stabilize orientation along the blade axis.

Therefore, if the tip-satellite is very small, the possibility exists for extremely low areal densities, in the unlimited case, approaching the areal density of the film itself.

The UltraSail concept was derived from the Heliogyro. The Heliogyro concept was developed in 1967-1968 by Richard H. MacNeal. ${ }^{3,4}$ The Heliogyro's configuration resembled a helicopter in that there were multiple long blades attached to a center hub. The pitch of the blades was controlled at the hub. The blades were deployed using centrifugal force, hence high spin rates were needed for deployment. These high spin rates were also needed to keep the blades reasonably flat against the solar photon pressure. The major difference between the Heliogyro and UltraSail is the addition of the aforementioned tip-satellites attached to the end of the blades. The advantage of these tip satellites is the elimination of the need for the high spin rates needed for the Heliogyro concept.

UltraSail could potentially accomplish numerous missions. Mission scenarios include missions to Mercury, which traditionally have high energy requirements. ${ }^{5,6,7}$ Solar polar orbits are also missions with high energy requirements, but are becoming increasingly possible with current solar sail technology. ${ }^{8}$ Missions to one or more near-Earth asteroids have also been proposed., ${ }^{9,10,11}$ Missions such as these, especially those with multiple objectives, are more and more attainable, as the high energy requirements are less of an obstacle to a solar sail mission. In addition, missions to the outer solar system and solar system escape have also been studied. ${ }^{12,13,14}$ Other missions unique to solar sails are the non-Keplerian orbits that are impossible to achieve with other spacecraft due to the high propellant mass requirements. ${ }^{15,16}$ These non-Keplerian missions include new Sun-planet-sail solutions to the three-body problem that are a function of sail parameters and attitude.

For the following analysis, it was assumed that the UltraSail system orbited the Sun at $1 \mathrm{AU}$. This assumption made it easier to illustrate the basic dynamic and control principles of the system. This simplification alleviated the need to integrate a changing solar pressure over various distances from the Sun, but could easily be approximated by the inverse square law.

\section{Solar Radiation Pressure}

A right-handed orthogonal coordinate system was constructed by defining the $z$-axis to coincide with the spin axis of the UltraSail. Positive $z$ was defined to be in the direction away from the sun. The $x$-axis was defined to be normal to the $z$-axis in the direction of an undeflected blade. Note that this coordinate system rotates with the blade. 
Because the rotating blades are not rigidly attached to the hub, they will be deflected away from the Sun due to the photon force until an equilibrium is achieved between centrifugal force and photon force. In this analysis, the blades were assumed to remain straight, even though in reality they will assume a catenary shape of the form:

$$
z(x)=-\frac{P_{o}}{\omega^{2}}\left[\frac{c x_{m} \operatorname{arctanh}\left[x\left(\rho c /\left(2 m_{s a t} R+\rho c R^{2}\right)\right)^{1 / 2}\right]}{\left[\rho c R / 2\left(m_{s a t}+\rho c R / 2\right)\right]^{1 / 2}}+\frac{\ln \left[\omega^{2}\left(m_{s a t} R+\rho c R^{2} / 2-\rho c x^{2} / 2\right)\right]}{\rho}\right]
$$

where $x_{m}=R\left[1-m_{\text {sat }} /\left(M / N+m_{\text {sat }}\right)\right]$. The above equation gives deflection in the $z$ direction as a function of the $x$ coordinate of the point along the centerline of the sail. For small deflection angles, the pressure on a catenary blade shape versus a straight blade will be nearly identical. The necessity to display maximum possible solar sail area to the Sun precludes large deflection angles, and thus the straight blade assumption is valid (Figure 2).

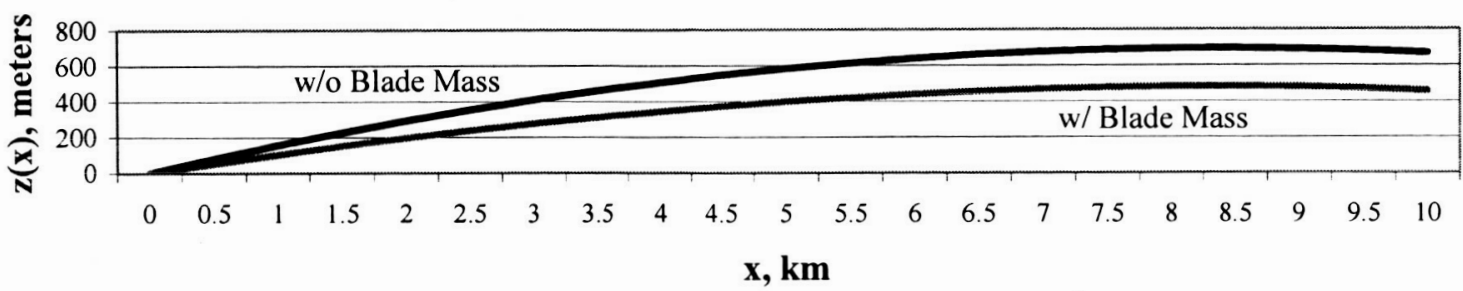

Figure 2. Catenary Shape

In the UltraSail design, the amount of this deflection can be controlled by the angular velocity of the blades (at higher angular velocities, the deflection angle $(\zeta)$ will decrease due to higher centrifugal force). As will be discussed later, part of this work involved finding the amount of centrifugal force that yielded the optimal deflection angle, based on increased or decreased fuel consumption of the tip-satellites and the associated changes in tipsatellite mass.

The deflection angle, $\zeta$, can be described relative to a reference plane that is defined by the blades in force free space. The total normal and transverse force exerted on a solar sail is given by: ${ }^{15}$

$$
\begin{gathered}
F_{n}=P A\left\{(1+\tilde{r} s) \cos ^{2} \zeta+B_{f}(1-s) \tilde{r} \cos \zeta+(1-\tilde{r}) \frac{\varepsilon_{f} B_{f}-\varepsilon_{b} B_{b}}{\varepsilon_{f}+\varepsilon_{b}} \cos \zeta\right\} \mathbf{n} \\
F_{t}=P A(1+\tilde{r} s) \cos \zeta \sin \zeta \mathbf{t}
\end{gathered}
$$

The first term in $F_{n}$ is the force due to absorbed and specularly reflected photons, the second term is due to the fact that in real sail material, some portion of the photons are non-specularly reflected, and the last term arises due to the thermal re-emission of photons from the front and back surfaces of the sail. In $F_{t}$ the only term comes from absorbed and specularly reflected photons.

Because the blades will always experience some deflection, it is more useful to look at the force in the $z$ direction, instead of dealing with directions that will change as the deflection changes. This force can be written as:

$$
F_{z}=P A\left\{(1-\tilde{r} s) \cos \zeta+2 \tilde{r} s \cos ^{3} \zeta+B_{f}(1-s) \tilde{r} \cos \zeta+(1-\tilde{r}) \frac{\varepsilon_{f} B_{f}-\varepsilon_{b} B_{b}}{\varepsilon_{f}+\varepsilon_{b}} \cos \zeta\right\} \mathbf{z}
$$




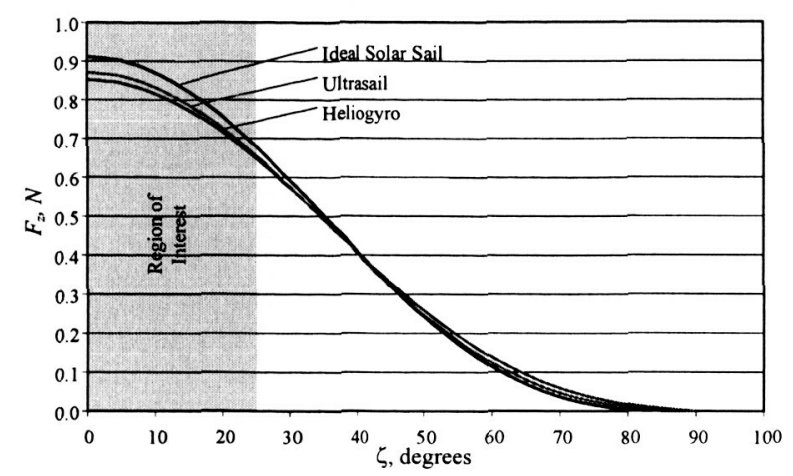

Figure 3. $F_{z}$ vs. $\zeta$.

Table 1. Solar Sail Film Properties

\begin{tabular}{lcccccc}
\hline & $\tilde{r}$ & $s$ & $\varepsilon_{f}$ & $\varepsilon_{b}$ & $B_{f}$ & $B_{b}$ \\
\hline Ideal Sail & 1 & 1 & 0 & 0 & $2 / 3$ & $2 / 3$ \\
Heliogyro & 0.88 & 0.94 & 0.05 & 0.55 & 0.79 & 0.55 \\
UltraSail & 0.91 & 0.94 & 0.03 & 0.27 & 0.79 & 0.55 \\
\hline
\end{tabular}

The optical constants used in the equations above are listed for several different types of sails in Table 1 and the resulting $z$-direction force is graphed in Figure 3. SRS Technologies provided the reflectivity, specular reflectivity and the emissivities for their 2.5 micron sail material. Lambertian properties were not available for the SRS sail material, so the non-ideal values for Heliogyro were assumed. Note that the deviation from the ideal force is less than $10 \%$ for both the Heliogyro and SRS films.

\section{Dynamics, Control, and the Force Ratio}

As with all solar sails, the normal of the sail points in the direction of acceleration. The blades of the UltraSail are manipulated to control this direction. One method of changing the acceleration vector is to change the orbital plane of the tip satellites. If it is assumed that the blades follow the tip satellite trajectory, then changing the inclination of the tip satellite will change the direction of the normal of the blade. The orbit of the tip satellite was considered to have a defined orbit radius of the length of a single blade. The tip velocity, or circular, velocity $\left(V_{t i p}\right)$ of the tip satellite can be found by multiplying the angular velocity by the length of the blade. The $\Delta v$ of one tip satellite for a single plane change can then be found using:

$$
\Delta V=2 V_{t i p} \sin \left(\frac{\Delta i}{2}\right)
$$

where $\Delta i$ is the desired inclination change.

When orbiting the Sun, it is desired that the spin axis is parallel to the Sun-spacecraft line. This pointing ensures the maximum sail area is presented to the Sun over the course of an orbit. Therefore, precession of the spin axis is required. If an UltraSail was orbiting at a given distance from the Sun, the spin axis would be precessed every $n$ number of days to ensure the sail normal is pointed away from the Sun. Figure 4 depicts the UltraSail's precession. 


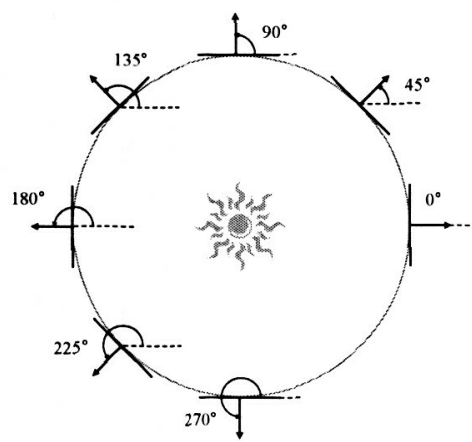

Figure 4. Precession of the Spin Axis.

The total $\Delta V$ required for a single blade over an entire UltraSail mission can be found using Eq. 5 . To simplify the calculation, a small angle approximation can be used. Then the equation is integrated over the entire angular change. This total $\Delta V$ required for one tip satellite was found to be:

$$
\Delta V=\int_{0}^{2 \pi k} 2 V_{t i p} \frac{d i}{2}=2 \pi k V_{t i p}
$$

where $k$ is the number of revolutions around the Sun. One result of this equation shows that no matter how many times the spin axis is precessed in one orbit (e.g. every 10 days, 20 days, etc.), the average $\Delta V$ for the entire orbit is the same, as long as the number of days does not exceed the small angle approximation. The amount of time between precessions is determined directly from the desired pointing accuracy of the spin axis.

An important parameter to characterize the rotation speed of the UltraSail was found to be the force ratio (FR). The force ratio was defined to be ratio of the centrifugal force on the blade/tip-satellite system and the force due to solar pressure. The purpose of the following study was to determine how the force ratio (FR) affects the equilibrium deflection angle $(\zeta)$ of an UltraSail blade. Increasing the force ratio decreases the deflection angle of the blades, which is desirable to maximize the solar sail area pointing towards the Sun. However, increasing the force ratio increases the angular velocity of the UltraSail, thereby increasing the propellant needed to maneuver the tip satellites. The goal of this analysis was to determine the minimum force ratio that results in an acceptable deflection angle.

There were two major forces considered when performing this analysis. The solar pressure will cause the blades to deflect away from the Sun (increasing $\zeta$ ), while the centrifugal force will cause the blades to deflect towards the Sun (decreasing $\zeta$ ). The two forces balance out to some final deflection angle. The problem was set up such that an initial tip velocity was found as a function of force ratio. Assuming the tip satellite moves in a circular motion allows the simple equation for centrifugal acceleration for circular motion to be used. From this equation, a force due to this centrifugal acceleration was found as a function of tip velocity. The expression for the velocity of the tip satellite was found to be:

$$
V_{t i p}=\sqrt{\frac{L^{2} c(F R) P_{o}}{m_{e q}}}
$$

where $m_{e q}=m_{\text {sat }}+\frac{1}{2} m_{\text {blade }}$. The angular velocity could then be expressed as:

$$
\omega=\frac{V_{t i p}}{L \cos (\zeta)}
$$

where $V_{t i p}$ is the velocity of the tip satellite. 
When the angular velocity of the UltraSail is set, there is an associated deflection angle that corresponds to this angular velocity due to the fact that the solar pressure will cause the blades to deflect away from the Sun. The moment due to centrifugal acceleration and the moment due to solar pressure (about the $y$-direction) for a perfectly reflecting solar sail were set equal to each other, and an expression for $\omega$ as a function of $\zeta$ was found. This expression can be found in Eq. 9.

$$
\omega^{2}=\frac{3 c P_{o} \cos ^{2}(\gamma-\zeta)}{2\left(3 m_{\text {sat }}+m_{\text {blade }}\right) \cos (\zeta) \sin (\zeta)}
$$

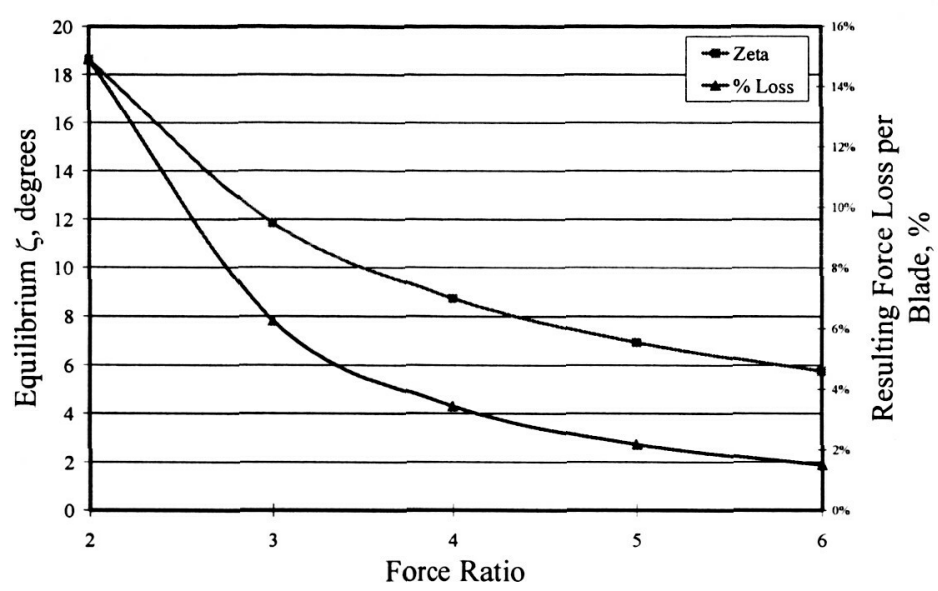

Figure 5. Equilibrium $\zeta$ vs. Force Ratio.

The variable $\gamma$ was defined as the angle between the Sun-spacecraft line and the spin axis. In this expression, it was also assumed that the there was no twist of the blades $(\theta=0)$.

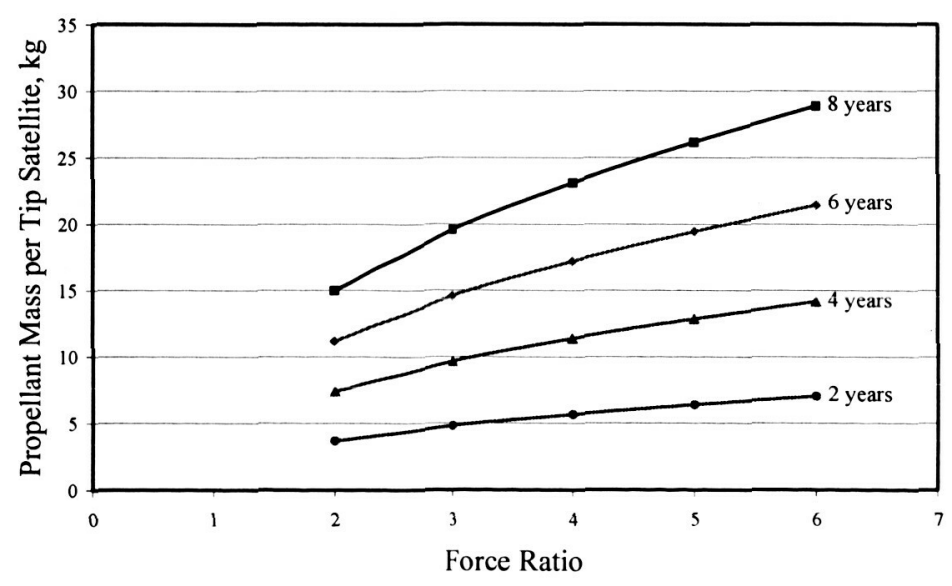

Figure 6. Propellant Mass per Tip-Satellite vs. Force Ratio.

In this analysis, the change in propellant was taken into account as the $V_{t i p}$ changed. Once the $V_{t i p}$ was found, the required $\Delta V$ for was found to maintain this $V_{t i p}$. The tank mass of the tip satellite was then altered, which also affects the equilibrium $\zeta$ due to the fact that the equilibrium relation is a function of $m_{s a t}$. The change in the tip satellite mass could then be approximated and a new angular velocity could be found. The angular velocity was 
used in Eq. 9 to numerically determine the new equilibrium $\zeta$. Due to the fact that the equilibrium $\zeta$ changed, a new centrifugal force was found for the given force ratio. Then a new $V_{\text {tip }}$ was found and the equations were cycled until the equilibrium $\zeta$ converged.

The results of this analysis produced several interesting results. Figure 5 illustrates how the equilibrium $\zeta$ decreased as a function of force ratio for a two year mission. The left side of this plot depicts corresponding force due to solar pressure loss (for one blade) as the FR increases. The equilibrium $\zeta$ was found to decrease from about $16^{\circ}$ as the force ratio increased from 2 to 6 . It is interesting to note that the increasing the force ratio from 2 to 3 produces the most decrease of the equilibrium $\zeta$, while after increasing the force ratio from 4 to 6 produces little gain.

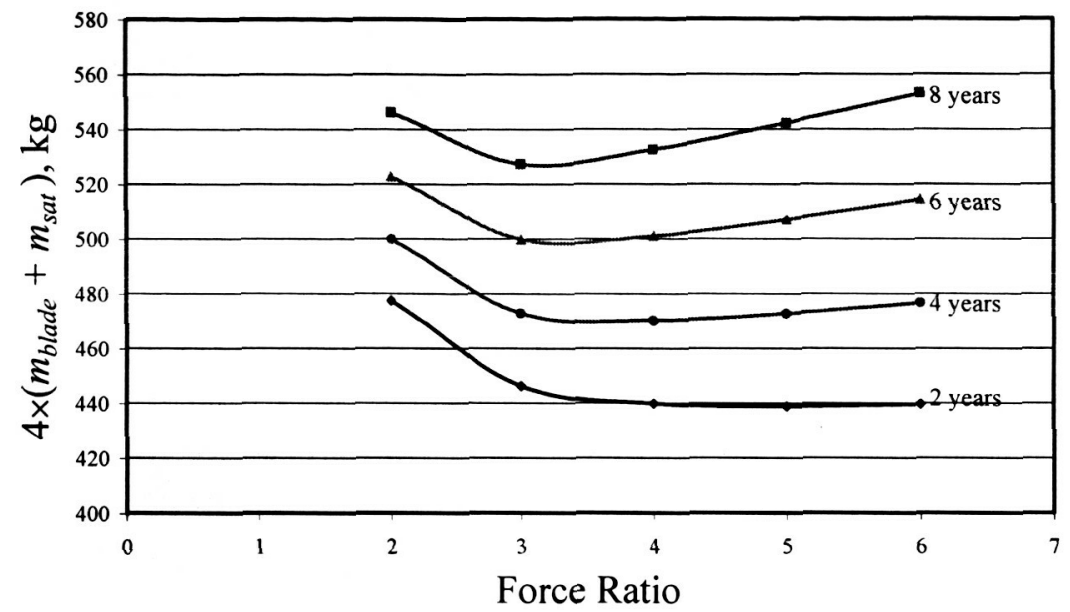

Figure 7. Mass of all Tip-Satellites and Blades vs. Force Ratio for 4 Mission Durations.

Figure 6 characterizes the propellant mass for one tip satellite as the force ratio increases for varying mission lengths. The slope of the lines increase as the mission length increases because the mass of the propellant and tank were changed as the force ratio was increased. As mission length increased, the required tank mass grew as the force ratio was increased, therefore even more propellant was needed to keep the force ratio constant.

The final part of this analysis was to determine the most appropriate force ratio for a given mission length. The force due to solar pressure for the baseline UltraSail design when the deflection angle $\zeta$ is zero was found to be $0.228 \mathrm{~N}$. This value represents the maximum possible force that the UltraSail system can experience. Then, for combination force ratios and mission length, the blade length was increased until the solar pressure reached this maximum. For large deflection angles, (low force ratio) the blade length needed to be increased more, as the force drops with the cosine of the deflection angle. For each new blade length, and hence increased blade mass, the angular velocity of the system was assumed to remain the same. Because blade length increased and angular velocity remained the same, the tip velocity increased. This change required a new value for the amount of propellant and the tank mass. From this, a new, greater, tip-sat mass was calculated and the problem repeated until it converged. These increases were summed for all four satellites and blades.

The total mass of the four tip satellites and blades were plotted as a function of force ratio for varying mission lengths (Figure 7). To maximize the payload, the blade and tip-sat system mass must be minimized. From this figure, there clearly exists a force ratio at which a minimum tip-satellite and blade mass occurs. This optimum force ratio runs between 3 and 3.5 for the higher mission lengths. For the two year mission, the minimum is at a force ratio around 5. However, the payload mass gain is so minimal between 3.5 and 5 that it does not seem necessary to operate the UltraSail at higher force ratios. For the baseline Ultrasail design, a force ratio of 3 was adopted.

\section{Tip-Satellite and Hub Metrology}

The positions of the blades need to be monitored very closely to ensure ideal orientation with respect to the Sun. Since the ends of the blades are fixed to the deployment arm and tip-satellite, knowing the hub and tip-satellite orientations and positions should provide all of the necessary information to determine the orientation and shape of the blade. It was hoped that studies of formation flying satellites and how they communicate and determine position 
with respect to each other could be adapted to the UltraSail system. These types of systems would be ideal for the UltraSail, as the tip-satellites are on the order of several kilometers from the hub and one another.

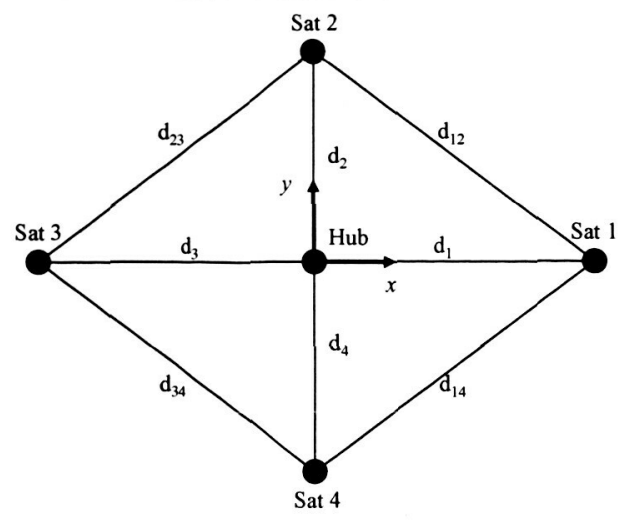

Figure 8. UltraSail Geometry.

The first problem involves determining position. It was thought that the easiest way to determine position would be to measure the distances between tip-satellites and hub, and tip-satellite to tip-satellite. These distances should produce some kind of nearly symmetric polyhedron, depending on the number of blades. For example, a four tipsatellite system should produce a five-sided shape, much like a flattened pyramid. The minimum number of distance measurements necessary to determine this volume, for a four-satellite system was found to be 9 . (See Figure 8) In fact for $N$ tip-sats, the minimum number of distances necessary, $D$, is given by $D=3(N-1)$ for $N>3$. A coordinate system was determined by tip-satellites 1 and 2 such that $\mathbf{r}_{1}=\left\{x_{1}, y_{1}, z_{1}\right\}=\left\{d_{1}, 0,0\right\}$ and $\mathbf{r}_{2}=\left\{x_{2}, y_{2}, z_{2}\right\}=\left\{d_{2} \cos \alpha_{12}, d_{2} \sin \alpha_{12}, 0\right\}$, where $\alpha_{12}=\cos ^{-1}\left[\left(d_{1}^{2}+d_{2}^{2}-d_{12}^{2}\right) / 2 d_{1} d_{2}\right]$. For any $i>3$ :

$$
\begin{aligned}
& \mathbf{r}_{\mathbf{i}}=\frac{1}{2\left(\mathbf{r}_{i-1} \times \mathbf{r}_{i-2}\right)^{2}}\{\left\{d_{i-2}^{2} d_{i-1}^{2}+\left(d_{i-1, i}^{2}-d_{i}^{2}\right) \mathbf{r}_{i-2} \cdot \mathbf{r}_{i-1}-d_{i-1}^{2}\left(d_{i-2, i}^{2}-d_{i}^{2}+\mathbf{r}_{i-2} \cdot \mathbf{r}_{i-1}\right)\right] \mathbf{r}_{i-2} \\
&\left.+\left[d_{i-2}^{2} d_{i-1}^{2}+\left(d_{i-2, i}^{2}-d_{i}^{2}\right) \mathbf{r}_{i-2} \cdot \mathbf{r}_{i-1}-d_{i-2}^{2}\left(d_{i-1, i}^{2}-d_{i}^{2}+\mathbf{r}_{i-2} \cdot \mathbf{r}_{i-1}\right)\right) \mathbf{r}_{i-1} \pm f\left(\mathbf{r}_{i-2}, \mathbf{r}_{i-1}\right)\left(\mathbf{r}_{i-2} \times \mathbf{r}_{i-1}\right)\right\} \\
& f\left(\mathbf{r}_{i-2}, \mathbf{r}_{i-1}\right)=\left\{\left(d_{i-1, i}^{2}-d_{i}^{2}\right)^{2} d_{i-2}^{2}-\left[\left(d_{i-2,1}^{2}-d_{i}^{2}\right)^{2}-2\left(d_{i-2, i}^{2}-d_{i}^{2}+d_{i-1, i}^{2}-d_{i}^{2}\right) d_{i-2}^{2}+d_{i-2}^{4}\right]\right. \\
&\left.-d_{i-2}^{2} d_{i-1}^{4}+2\left(d_{i-2, i}^{2}-d_{i}^{2}-d_{i-2}^{2}\right)\left(d_{i-1, i}^{2}-d_{i}^{2}-d_{i-1}^{2}\right) \mathbf{r}_{i-2} \cdot \mathbf{r}_{i-1}+4 d_{i}^{2}\left(\mathbf{r}_{i-2} \times \mathbf{r}_{i-1}\right)\right\}^{1 / 2}
\end{aligned}
$$

Once the shape is known, the dihedral angles are very easy to determine with basic geometric principles.

After the geometry of the system was determined, various distance measurement methods were studied. Three basic assumptions were made to simplify the first-order study. First, linear distances vary slowly enough to permit accurate results from a set of sequential measurements. Second, the sails themselves only minutely obstruct or disrupt the propagation of signals, such that a reasonable signal-to-noise ratio (SNR) can be maintained at each receptor. Lastly, enough onboard power would be available to transmit signals over nearly $10 \mathrm{~km}$ in free space, from one sail tip to the diametrically opposite one.

Due to the large distance from the Earth, GPS could not be used for positioning as it has been on many lowEarth orbiting satellites. Two techniques were initially suggested to achieve this goal: frequency difference measurements using a ramped-frequency generator and varying phase-shift comparator method. After closely evaluating the relative advantages of each, the latter ranging method was finally selected and further researched. Its basic premise relies on the phase difference between emitted and returned signals. These differences are linearly related to the spatial separation between the signal source and its distant target. In the case of the UltraSail spacecraft, the source would be the hub, and the target, one of the tip-sats. With prior knowledge of all satellite-tocentral spacecraft distances, inter-satellite ranges can be determined. An important source of concern was the presence of numerous $2 \pi$-multiples for each measured phase shifts. Circumventing this difficulty requires a repeated set of measurements at unrelated frequencies. Computer simulations demonstrated an approximately 10 -fold reduction in the uncertainty level with the addition of a second frequency, and nearly 100-fold with a third 
frequency. Extrapolation of these results provides confidence that precise ranging measurements could be made with a only small set of frequencies.

The distance measurements, while providing the positions of all tip-satellites and hub, only do so in a relative manner. That is to say, once the polyhedral shape is known from the distance measurements, its orientation in a space fixed frame is still not known. Therefore, Sun sensors and star trackers on every tip-satellite and hub, along with the assumption that the "pyramid" will open away from the Sun due to solar pressure, must be used to determine the space-fixed orientation of the entire system. Finally, these same sensors can be used to determine the attitude of the tip-satellites and hub.

\section{Tip-Satellite and Hub Design}

Design parameters for the UltraSail tip-satellite and hub subsystems are described here. The tip-satellite must perform several roles. First, its primary propulsion system will be responsible for unrolling the film from the mandrels. Next, it is required to spin up the system and maintain a fixed angular velocity, depending on the desired force ratio. As detailed above, the tip-satellites will have to carry a robust attitude and position determination system and the means to communicate with the hub. Finally, the attitude control system of the tip-satellites will be used to control the pitch of the blades.

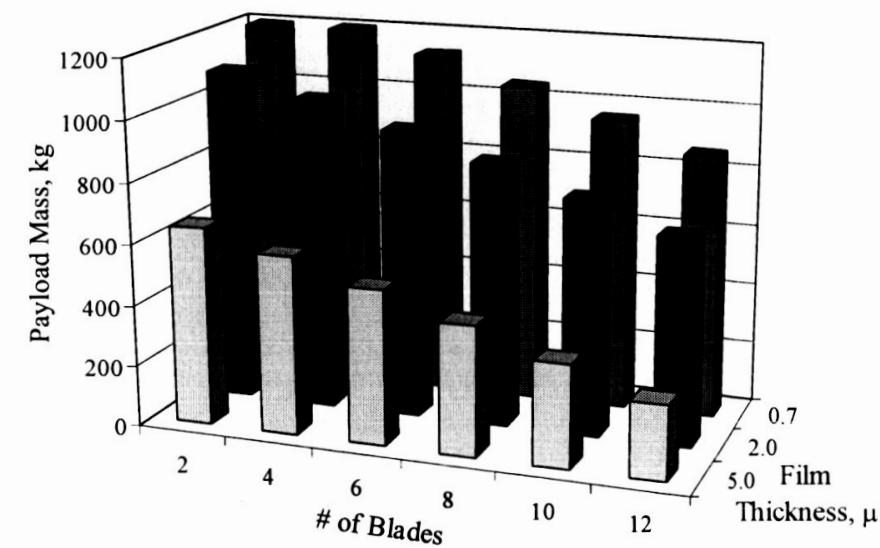

Figure 9. UltraSail Payload Comparison.
Table 2. UltraSail Baseline System Parameters.

\begin{tabular}{lr}
\hline Number of Blades & 4 \\
Mission Length, years & 2 \\
Photon force at 1 AU, N/m ${ }^{2}$ & $9.1 \mathrm{E}-06$ \\
Spin Force Ratio & 3.0 \\
CP-1 Film Density, $\mathrm{kg} / \mathrm{m}^{3}$ & 1430 \\
Sail Thickness, $\mathrm{m}$ & $2 \mathrm{E}-06$ \\
Total Sail Area, $\mathrm{km}^{2}$ & 1 \\
Total Sail Mass, $\mathrm{kg}$ & 286 \\
Characteristic velocity, $\mathrm{m} / \mathrm{s}$ & 37,378 \\
Blade Chord, $\mathrm{m}$ & 5 \\
Blade Length, $\mathrm{m}$ & 5000 \\
Launch Mass to $C_{3}=0, \mathrm{~kg}$ & 1541 \\
Tip-Satellite specific impulse, sec & 150 \\
Deployment Time, hrs & 10 \\
\hline
\end{tabular}

Mass analyses were conducted for the tip-sats, ${ }^{17}$ varying the number blades and film thicknesses, while holding force ratio fixed at 5 . The overall results are displayed in Figure 9 by showing the deliverable payload for the different combinations, assuming a Delta $2925 \mathrm{H}-9.5$ to $C_{3}=0$ launch mass of $1541 \mathrm{~kg}$. Launch mass to $C_{3}$ is a common method to compare the amount of payload mass that different rockets can deliver. The $C_{3}=0$ point is defined as the point in space where the spacecraft no longer has any hyperbolic excess velocity. The trend displayed in Figure 9 is obvious; there are extreme penalties associated with high blade number systems with a fixed sail area.

The baseline design chosen for detailed analysis was a four blade design, utilizing a 2 micron film thickness. This film thickness was chosen because it is more realistic in terms of what is technologically possible in the very near future. Key parameters are given in Table 2. Logically, to maximize areal density, it would make sense to minimize the number of blades, since there is a mass penalty for each additional blade in the form of an extra tipsatellite and an additional deployment arm mechanism. It was determined that for an UltraSail system, with a fixed surface area of $100,000 \mathrm{~m}^{2}$, and a realistic tip-satellite mass, that more than 8 blades prohibits a useful payload mass. High numbers of blades also increases potential deployment complications. Two blades appeared to be the ideal choice, since it is the minimum number of blades necessary for a symmetric system. For redundancy however, a four blade system was chosen for the baseline design, with the thought that if one blade were damaged, it and its partner could be jettisoned and the mission continued with two blades. The penalty for using four blades versus two was found to be approximately $80 \mathrm{~kg}$. 
The blades are CP-1 $2.0 \mu \mathrm{m}$ polyimide film with aluminum reflecting coating, supplied by SRS Techologies. They have Kevlar thread rip-stop and Kevlar edge reinforcement. The Kevlar serves to prevent tears in the film and to assume the pressure load. The mandrel on which the film is stored during launch is a perforated thin-walled aluminum or carbon-fiber core. The film is permanently attached to a deployment arm via a compliant attachment, which in turn is attached to the hub. The far side of the film is attached to the tip-satellite with the same compliant attachment. The arm is a motor driven deployable structure that allows the film roll to be moved from its stowed position to a distance far enough way from the hub and other rolls to prevent interference. The deployment sequence will involve spinning up the system with sail area parallel to the Sun-spacecraft line (no force on the sails) using the larger of the tip-sat thrusters. The centrifugal force generated by the spinning will unwind the film in a precisely controlled manner. Deployment time has been set at 10 hours, or $14 \mathrm{~cm} / \mathrm{s}$ film unwind speed. This speed is highly variable, and its determination is one focus of the experiment (See experiment section).

Subsystem mass analysis for the tip-sat and hub was conducted to provide baseline masses for the calculations performed above. Subsystems studied for the tip-satellites include propulsion, avionics, power, structure, and the blade deployment mechanism/compliant attachment.

The main structure of the tip-satellites consist of a simple truss, constructed of either aluminum or carbon fiber tubes. Size of the structure is $5 \mathrm{~m}$ long with a $0.5 \times 0.5 \mathrm{~m}$ cross section. The truss structure consists of 10 half meter cube elements, with cross members on each face of the cube. Using half inch carbon fiber tube with a $0.8 \mathrm{~mm}$ wall thickness for the truss, yields a mass of 4.6 kg. Fittings, attachment points, and passive thermal control systems bring the total structure mass up to $8.0 \mathrm{~kg}$.

The avionics system, as mentioned above, will be relatively complex because of the need for relatively decent positioning data. Therefore, each

Table 3. UltraSail System Masses, 4 Blades, $F R=3$, $2 \mathrm{~m}$ film thickness, 2 year mission length

\begin{tabular}{|c|c|c|}
\hline & TipSat (each) & Hub \\
\hline Propulsion Subsystem & 7.6 & 5.4 \\
\hline Avionics Subsystem & 6.2 & 14.2 \\
\hline Power Subsystem & 2.9 & 9.4 \\
\hline Structure & 8.0 & 7.7 \\
\hline Mass subtotal & 21.1 & 34.3 \\
\hline Contingency (30\%) & 6.3 & 11.0 \\
\hline Deployment arm & 10.7 & NA \\
\hline Subsystem Total & 41.7 & 47.7 \\
\hline Film & 71.5 & NA \\
\hline Sail System Mass & 113.2 & $\mathrm{NA}$ \\
\hline Total Mass & \multicolumn{2}{|c|}{500.5} \\
\hline Launch Mass to $\mathrm{C} 3=0$ & \multicolumn{2}{|c|}{1541} \\
\hline Usable Payload Mass & \multicolumn{2}{|c|}{1040.5} \\
\hline
\end{tabular}
tip-satellite will have a two Sun-sensors and two star-trackers, for redundancy. Further, the positioning information will be gathered with a microwave ranging system as described in the ranging section. Computation will be done at the hub in near real time to achieve nearly autonomous operation, so a modest communications system will be necessary to relay the information between hub and tip-satellite. This communications is also necessary for the relay of other pertinent subsystem data, commands, and state of health.

The power subsystem consists of body-mounted solar panels and associated power processing equipment. Beginning of life estimates for solar panel specific power were $50 \mathrm{~W} / \mathrm{kg}$. Power requirements for the tip-sat was assumed to be $50 \mathrm{~W}$, yielding $1 \mathrm{~kg}$ in solar arrays per satellite. Additional processing equipment and batteries were assumed to be another $2 \mathrm{~kg}$.

The propulsion system on the tip-sat will consist of 2 thruster units, supplied by Vacco Industries, mounted on either end of the truss structure, providing thrust on the order of $0.1 \mathrm{~N}$. These units utilize catalyzed Nitrous Oxide $\left(\mathrm{N}_{2} \mathrm{O}\right)$, to yield approximately $150 \mathrm{~s} \mathrm{I}_{\mathrm{sp}}$. The attitude control system will consist of nearly identical thrusters, also mounted on the ends of the truss and providing thrust on the order of $10 \mu \mathrm{N}$, using cold Butane. Because the mass analysis was conducted for three different film thicknesses, for different mission lengths and force ratios, the propellant masses varied widely (between 1 and $20 \mathrm{~kg}$ ). Therefore, the change in propellant system mass was analyzed. Assuming a pressure fed system, with a chamber pressure of 750 psi and Titanium tanks and using Ni's trade study for small satellite diaphragm propellant tanks, ${ }^{18}$ tank mass was determined as a function of initial propellant mass. Tankage masses ranged from $1-6 \mathrm{~kg}$.

Additionally, due to the preliminary nature of the design, a $30 \%$ mass contingency was added to the dry mass of the tip-sats and hub. The mass analysis for the hub was similar to that of the tip-sats, with subsystems masses listed in Table 3. It was shown that a tip-satellite dry mass of approximately $42 \mathrm{~kg}$ and a hub dry mass of just under $50 \mathrm{~kg}$ is possible with state of the art technology. This is encouraging from the standpoint of minimizing areal density of the combined blade and tip-sat system. 


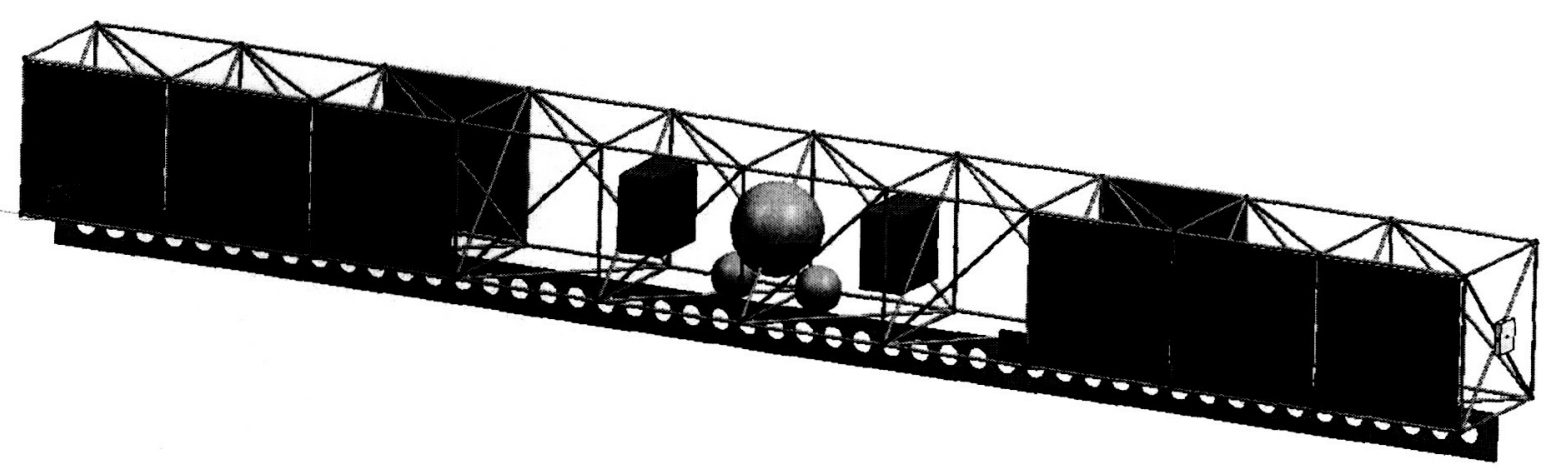

\section{Vacuum Deployment Experiment}

One of the most challenging aspects of the UltraSail project is the deployment of a potentially kilometers long sail from a rolled up configuration. The Vacuum Deployment Experiment (VDE) is designed to measure the required force to unwind the sail material from the mandrel in vacuum environment. Also, the experiment was designed to be portable enough to allow it to be placed on aboard a micro-gravity simulation aircraft. The unrolling can be studied in a zero-g environment in addition to the vacuum. Self-adhesion and electrostatic forces are expected to contribute the most resistance to unwinding. Further, the force as a function of unrolling speed needs to be determined. As seen in Figure 11, two reels will be placed in a vacuum chamber of our design. One reel is prewrapped with approximately 100 meters of $30 \mathrm{~cm}$ wide, 2.5 micron film from SRS technologies, and the other reel acts as a take up. The reels are synchronized by a timing belt, and connected to a precision variable speed motor on the outside of the tank by a ferro-fluidic rotary motion vacuum feedthrough.

The unwinding force will overcome the peeling force, which again is the sum of the electrostatic and adhesive forces between the sail surfaces. Figure 11 shows the partially constructed vacuum chamber and the Unigraphics 3D CAD model. The tension measurement system is shown in Figure 10. ${ }^{19}$

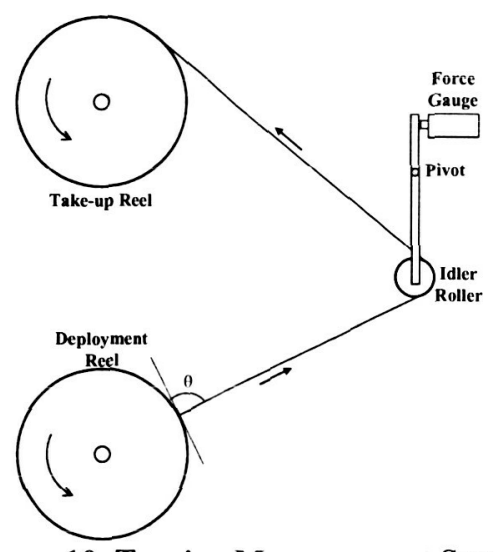

Figure 10. Tension Measurement System.

The force transducer shown in the picture will indirectly measure the tension in the sail material at different unwinding speeds. Additionally, the force transducer is accurate between $0.1 \mathrm{~g}$ and $125 \mathrm{~g}$. By closely measuring the angles of the film between the reels and the roller, along with the measured force, the film tension can be calculated. From By design of the experiment, the tension is equivalent to the peel force. Therefore, once the tension is known, Eq. 12 can be used to relate peel force to the force required to unwind the sail. ${ }^{20}$ 

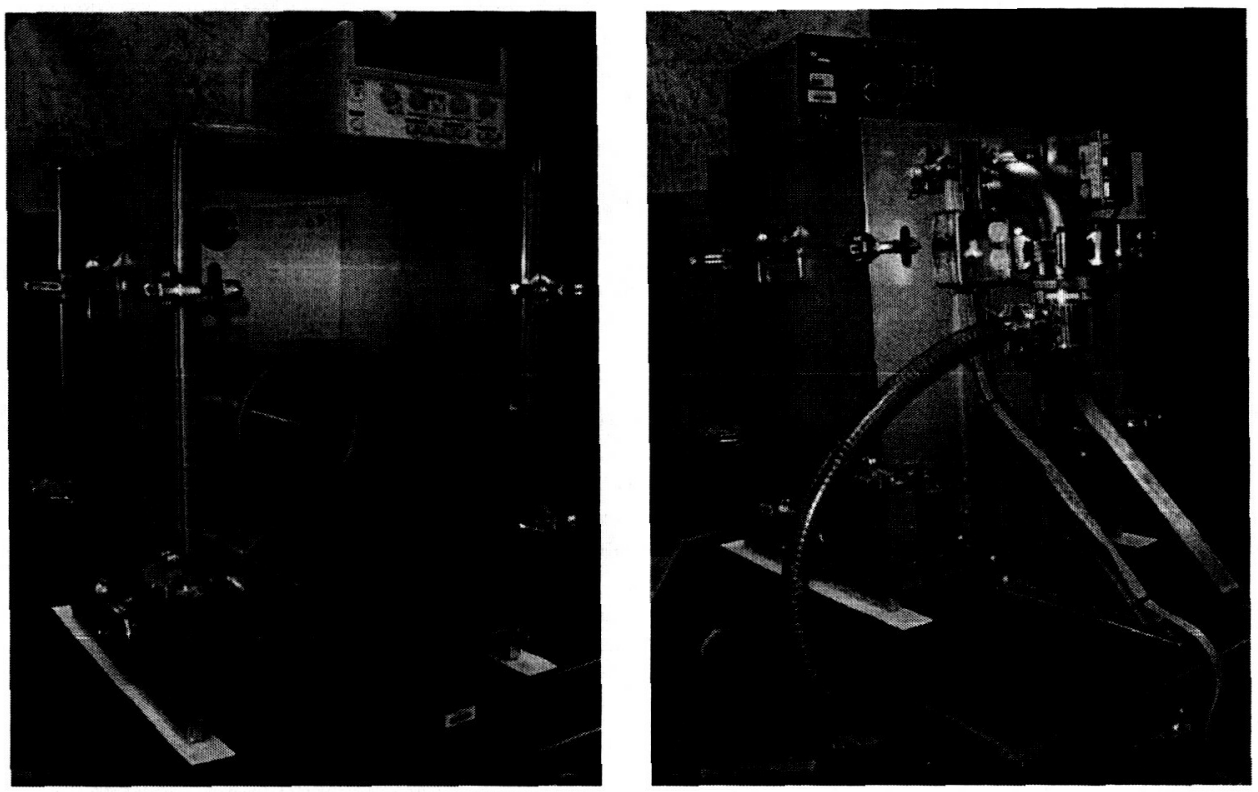

Figure 11. Vacuum Deployment Experiment (Front and Back)

$$
\left(\frac{F}{b}\right)^{2} \frac{1}{2 t E}+\left(\frac{F}{b}\right)(1-\cos \theta)-T=0
$$

where $F$ is the required unwinding force, $b$ is width of the film, $t$ is thickness of the film, $\theta$ is the peel angle, $E$ is the Young's modulus of the film, and $T$ is the peeling force. It is assumed that the moment of the reels is not a factor because they are synchronized by a motor driven timing belt, rather than being turned by the film itself. This is not the case with the idler roller, so extreme care was taken to ensure it was as lightweight and frictionless as possible. In this case, the first term of Eq. 12 can be neglected because of a large Young's Modulus and a small unwinding force. Therefore Eq. 12 becomes:

$$
\left(\frac{F}{b}\right)(1-\cos \theta)=T
$$

The peeling force $(T)$ in Eq. 13 is an unknown function of unwinding speed. The nature of this function is to be determined. The required unwinding force will be provided by tip satellites and/or by the centrifugal force. The expected tension range is very small (less than $0.01 \mathrm{~N}$ ), so the experiment is designed to measure these small forces. One of the methods to increase the measurable force is to change the pivot on the lever arm contacting the forcetransducer. The transducer will be biased by a constant force spring to allow measurement in the middle of its range, to further increase accuracy. Unwinding speeds up to $1 \mathrm{~m} / \mathrm{s}$ can be simulated by the experiment, which is equivalent to a minimum deployment time of 1.4 hours.

\section{Conclusion}

It has been shown that solar sails can compete with and in some missions outperform Solar Electric Propulsion systems in terms of delivered payload mass. Specifically, the UltraSail solar sailing concept achieves this by minimizing support structures through the use of spinning solar sails controlled by micro tip-satellites. Payload mass fractions were found to be as high as $2 / 3$ of delivered $\mathrm{S} / \mathrm{C}$ mass. Equivalent thrust and specific impulse of UltraSail was estimated and will be published in future work. Continuing work on this project includes the study of cyclic pitching of the blades to precess the angular velocity vector. By changing the angle of incidence of some 
blades for a fraction of the system revolution period, preliminary calculations have shown that this may be a viable way to "steer" the UltraSail system. The vacuum deployment experiment will determine an important characteristic of the SRS supplied film material: the force required to deploy the material as a function unwinding speed. This type of experiment is critical to demonstrate the feasibility of unrolling a stowed kilometer scale sail in space.

\section{Acknowledgments}

This work was funded by NASA Contract \# NNM04AB18C. We wish to thank the In-Space Propulsion Technology Program technical monitors Joseph Bonometti and John Dankanich, along with Kent Elam, head of the Theoretical and Applied Mechanics machine shop, and Tim Prunkard, head of the Civil Engineering machine shop.

\section{References}

\footnotetext{
${ }^{1} J o h n s o n, \quad L$, et al., “NASA's In-Space Propulsion Technology Program: Overview and Update," $40^{\text {th }}$ AIAA/ASME/SAE/ASEE Joint Propulsion Conference and Exhibit, AIAA Paper 2004-3841, Fort Lauderdale, Florida, July 1114, 2004.

${ }^{2}$ Montgomery, E., and Johnson, L., "The Development of Solar Sail Propulsion for NASA Science Missions to the Inner Solar System," $45^{\text {th }}$ AIAA/ASME/ASCE/AHS/ASC Structures, Structural Dynamics \& Materials Conference, AIAA Paper 20041506, Palm Springs, California, April 19-22. 2004.

${ }^{3}$ MacNeal, R. H., “The Heliogyro, An Introductory Flying Machine,” NASA Contractor's Report CR 84460, June 1967.

${ }^{4}$ MacNeal, R. H., "Structural Dynamics of the Heliogyro," NASA CR-17445A, 1971.

${ }^{5}$ Leipold, M., Borg, E., Lingner, S., Pabsch, A., Sachs, R., Seboldt, W., "Mercury Orbiter with a Solar Sail Spacecraft," Acta Astronautica, Vol. 35, Suppl., pp. 635-644, 1995.

${ }^{6}$ Leipold M., Seboldt, W., Lingner, S., Borg, E., Herrmann, A., Pabsch, A., Wagner, O., Bruckner, J., "Mercury SunSynchronous Polar Orbiter with a Solar Sail," Acta Astronautica, Vol. 39, No. 1-4, pp. 143-151, 1996.

${ }_{7}^{7}$ Macdonald, M., McInnes, C., "Solar Sail Capture Trajectories at Mercury", AIAA/AAS Astrodynamics Specialist Conference and Exhibit, AIAA Paper 2002-4990, Monterey, California, August 5-8, 2002.

${ }^{8}$ Sauer, C. G., "Solar Sail Trajectories for Solar Polar and Interstellar Probe Missions," AIAA/AAS Astrodynamics Specialist Conference, AIAA Paper 99-336, Girdwood, Alaska, August 16-19, 1999.

${ }^{9}$ Pomazanov, M. V., "Solar Sail: Principles of Construction, Control, and Flights to Asteroids," Cosmic Research, Vol. 37, No. 4, pp. 374-381, 1999.

${ }^{10}$ Morrow, E., Scheeres, D. J., Lubin, D., "Solar Sail Orbit Operations at Asteroids," Journal of Spacecraft and Rockets, Vol. 38, No. 2, March-April 2001.

${ }^{11}$ Hughes, G. W., McInnes, C. R., "Small-Body Encounters Using Solar Sail Propulsion," Journal of Spacecraft and Rockets, Vol. 41, No. 1, January-February 2004.

${ }^{12}$ Leipold, M., "Solar Sail Technology Development and Application to Fast Missions to the Outer Heliosphere," American Institute of Physics Conference Proceedings, Vol. 552. Issue 1, pp. 385-392, February 2001.

${ }^{13}$ Sharma, D. N., Scheeres, D. J., "Solar-System Escape Trajectories Using Solar Sails," Journal of Spacecraft and Rockets, Vol. 41, No. 4, July-August 2004.

${ }^{14}$ Dachwald, B., "Optimal Solar Sail Trajectories for Missions to the Outer Solar System", AIAA/AAS Astrodynamics Specialist Conference and Exhibit, AIAA Paper 2004-5406, Providence, Rhode Island, July 16-19, 2004.

${ }^{15}$ McInnes, C.R., Solar Sailing: Technology, Dynamics, and Mission Applications, Springer-Praxis, Chichester, UK, 1999.

${ }^{16}$ Hughes, G. W., McInnes, C. R., "Solar Sail Hybrid Trajectory Optimization for Non-Keplerian Orbit Transfers," Journal of Guidance, Control, and Dynamics, Vol. 25, No. 3, pp. 602-604, 2002.

${ }^{17}$ Wertz, J.R. and Larson, W.J., Space Mission Analysis and Design, $3^{\text {rd }}$ Ed., Microcosm, El Segundo, CA, 1999.

${ }^{18}$ Ni, M.S., Willmes, G.F., Burton, R.L., and Osborn, M.F., "Propulsion Trade Study for Small Satellites," Technical Report UILU ENG-96-506, University of Illinois at Urbana-Champaign, November 28, 1995.

${ }^{19}$ Hawkins, W. E., The Plastic Film and Foil Web Handling Guide, CRC Press, London, 2003, Chaps. 2, 3.

${ }^{20}$ Kendall, K., "Thin-film Peeling: The Elastic Term," J. Phys. D:Appl. Phys., Vol. 8, 1975.
} 